\title{
Ovarian transposition
}

\author{
Elisabeth Reiser · Bettina Böttcher · Danijela Minasch · Julian Mangesius · Bettina Toth
}

Received: 31 May 2020 / Accepted: 18 August 2020 / Published online: 18 September 2020

(c) The Author(s) 2020

\begin{abstract}
Summary Cytotoxic chemotherapy regimens and radiotherapy can lead to acute ovarian failure, premature ovarian insufficiency and menopause. Fertility preservation options before radiotherapy include ovarian transposition, where one or both ovaries are placed outside the radiation field. However, the efficacy of ovarian transposition is questioned, as the conservation of ovarian function varies between 17 and $95 \%$ in the literature.
\end{abstract}

Keywords Fertility preservation - Ovarian function . Radiotherapy $\cdot$ Gonadotoxic therapy $\cdot$ Surgery

\section{Introduction}

Pelvic radiotherapy is a standardized treatment in gynecologic, anal or rectal cancer as well as Hodgkin lymphoma [1, 2]. It disturbs gonadal function and can cause primary ovarian insufficiency (POI) ending up in infertility [3]. The extent of damage to ovarian function depends on several factors: patient age, radiation dosage, radiation field and possible combination with chemotherapy. In the case of pregnancy after cancer treatment, the risks of miscarriage, stillbirth, preterm birth and abnormal placentation may be increased [4, 5]. As survival rates of cancer patients are constantly increasing, fertility sparing is of growing interest and importance. More than $75 \%$ of patients suffering from

E. Reiser $(\bowtie) \cdot$ B. Böttcher $\cdot$ B. Toth

Department of Gynecological Endocrinology and Reproductive Medicine, Medical University of Innsbruck, Anichstraße 35, 6020 Innsbruck, Austria elisabeth.reiser@i-med.ac.at

\section{Minasch $\cdot$ J. Mangesius}

Department of Therapeutic Radiology and Oncology,

Medical University of Innsbruck, Innsbruck, Austria cancer need to be counseled on fertility preservation options as they wish to have children in the future [6].

Fertility preservation options before radiotherapy include ovarian transposition (OT), cryopreservation of ovarian tissue and/or ovarian stimulation with cryopreservation of (fertilized) oocytes. The different techniques can also be combined depending on patient age, planned radiotherapy and the necessity of chemotherapy.

\section{Surgical procedure}

Ovarian transposition was first established in 1952 by Batten to reduce the exposure of the female gonads to high radiation doses [7]. Mainly by laparoscopy, one or both ovaries are (temporarily) positioned at least $2 \mathrm{~cm}$ above the pelvic brim. After ligating the Fallopian tube and the ovarian ligament, the ovary is attached to the abdominal wall. Both ovaries are marked with titanium metallic clips to determine their exact position in future controls. The achieved distance to the radiation field is of great importance, as in a $10-\mathrm{cm}$ distance there is still $10 \%$ of radiation dosage active [8]. In a multivariate analysis, the position of the fixed ovary was the greatest prognostic factor for preservation of ovarian function [9]. The risks of the procedure itself are described as low and mainly consist of the standard risks of laparoscopy. Ovarian cysts can occur, but are more likely a sign of impaired ovarian function and are self-limited.

However, by leaving the ovaries in situ, ovarian metastases might be overlooked. This depends on the type of cancer: the risk for ovarian metastases of cervical cancer (adenocarcinoma and squamous cell carcinoma) is $1.7 \%$ and $0.5 \%$, respectively [10]. The risk for "port site metastasis" due to the laparoscopic approach is described as less than $1 \%$ [11]. 
The benefit of ovarian transposition in terms of fertility preservation has been widely and controversially discussed. In general, only small numbers of participants were included (eight to 107) in existing studies $[12,13]$. The analyses of the remaining ovarian function ranges from only assessment of climacteric complaints to the combination of regular menses, laboratory values, climacteric complaints, imaging and/or pregnancy.

\section{A matter of age}

Costa-Roig et al. reported on 21 OT in 13 patients (aged between 10 and 15 years) without any postoperative complications in 10 years of experience in a pediatric cancer center. During the follow-up period (between 12 months and 10 years), four patients died due to progression of disease. Of the remaining nine patients, six completed oncologic treatment. Three of these six patients showed regular menses after cancer treatment [14]. Furthermore, the effect of OT on ovarian preservation was reviewed in 49 longterm survivors of childhood Hodgkin lymphoma (HL) that underwent OT before radiotherapy and compared to $41 \mathrm{HL}$ patients without OT [15]. The median age at OT was 15 years (range: 4-24 years), and the median age at evaluation was 38 years (range: 25-60 years). POI was defined in individuals under 40 years with amenorrhea $>6$ months and plasma estradiol (E2) levels $<17 \mathrm{pg} / \mathrm{mL}$ as well as follicle stimulating hormone $(\mathrm{FSH}) \geq 30 \mathrm{IU} / \mathrm{L}$. There was no significant association between OT and occurrence of POI. However, higher pelvic radiation doses and higher doses of alkylating agents for chemotherapy were associated with an increased risk of POI. Nevertheless, the probability of a first pregnancy did not differ between the OT and the non-OT group. This study with a large group of patients and a long period of follow-up indicates that OT may not prevent radiation-induced ovarian damage. Of note, radiation techniques have changed over time (study performed 1962-2005) and dosimetry calculations were not included. In a cohort of older patients (mean age at transposition, 33.4 years) the positive effect of OT on ovarian survival was shown in all age groups (25-30, 30-35 and 35-40) compared to women without OT. However, ovarian survival rates decreased with increasing age of patients at time of OT [16].

\section{A matter of dose}

Wallace et al. calculated the likelihood of sterility depending on radiation dose measured by the effective sterilizing dose (ESD=dose after which the patients' primordial oocyte population will fall below 1000). The ESD decreased with age and was estimated as 18.4 Gy at 10 years, approximately $14.3 \mathrm{~Gy}$ at 30 years and only 6 Gy over 40 years [17]. The estimated dose to destroy $50 \%$ of primordial follicles (LD50) was described as less than $2 \mathrm{~Gy}$ [18]. Both counseling the patient and calculating the radiation field depend on this mathematical model, which was established over 15 years ago and has never been questioned since. Moreover, these data were collected long before the availability of high-resolution imaging.

Yin et al. investigated the influence of radiation dose on ovarian function after OT in cervical cancer patients [19]. Of 118 patients (age: 24-49 years), 105 received a limited radiation dose (as low as possible) to the ovaries, whereas the rest received the full dose. During follow-up, ovarian function was evaluated by measuring levels of FSH and E2 1 year after completion of pelvic irradiation. A total of 41 of the 105 patients $(39.0 \%)$ that underwent intensity-modulated radiotherapy with limited ovarian dose preserved their normal ovarian function, whereas none of the 13 patients without dose reduction preserved their ovarian function.

OT is one option of fertility preservation before radiation therapy, but patients need to be informed about the limits of existing data and limits of this technique itself. Patients should be carefully selected: menopausal women and women aged over 40 years are ideal candidates for OT. Moreover, patients with cancers at moderate or high risk for ovarian metastasis may also not be good candidates [20]. The success rate and risks need to be discussed with the patient.

\section{Take home message}

1. Radiation-induced ovarian damage depends on patient age, radiation dosage/field and combination with chemotherapy.

2. OT can be offered before radiation; nevertheless, the benefit in terms of fertility preservation is a matter of debate.

Funding Open access funding provided by University of Innsbruck and Medical University of Innsbruck.

Conflict of interest E. Reiser, B. Böttcher, D. Minasch, J. Mangesius, and $\mathrm{B}$. Toth declare that they have no competing interests.

Open Access This article is licensed under a Creative Commons Attribution 4.0 International License, which permits use, sharing, adaptation, distribution and reproduction in any medium or format, as long as you give appropriate credit to the original author(s) and the source, provide a link to the Creative Commons licence, and indicate if changes were made. The images or other third party material in this article are included in the article's Creative Commons licence, unless indicated otherwise in a credit line to the material. If material is not included in the article's Creative Commons licence and your intended use is not permitted by statutory regulation or exceeds the permitted use, you will need to obtain permission directly from the copyright holder. To view a copy of this licence, visit http://creativecommons.org/licenses/by/4.0/. 


\section{References}

1. Sedlis A, et al. A randomized trial of pelvic radiation therapy versus no further therapy in selected patients with stage IB carcinoma of the cervix after radical hysterectomy and pelvic lymphadenectomy: A Gynecologic Oncology Group Study. GynecolOncol. 1999;73(2):177-83.

2. Folkesson J, et al. Swedish rectal cancer trial: long lasting benefits from radiotherapy on survival and local recurrence rate. JClin Oncol. 2005;23(24):5644-50.

3. Davies MC, Hall ML, Jacobs HS. Bone mineral loss in young women with amenorrhoea. BMJ. 1990;301(6755):790-3.

4. TehWT, etal. Theimpact of uterineradiation on subsequent fertility and pregnancy outcomes. Biomed Res Int. 2014; https://doi.org/10.1155/2014/482968.

5. ThibaudE, et al. Ovarian function after bone marrow transplantation during childhood. Bone Marrow Transplant. 1998;21(3):287-90.

6. Schover LR, et al. Having children after cancer. Cancer 1999;86(4):697-709.

7. Batten R, Brown DE. Protection of ovaries from radiation. Lancet. 1956;270(6929):939-40.

8. Winarto $\mathrm{H}$, et al. The need for laparoscopic ovarian transposition in young patients with cervical cancer undergoing radiotherapy. Int J Reprod Med. 2013; https://doi.org/10. $1155 / 2013 / 173568$.

9. Hwang $\mathrm{JH}$, et al. Association between the location of transposed ovary and ovarian function in patients with uterine cervical cancer treated with (postoperative or primary) pelvic radiotherapy. Fertil Steril. 2012;97(6):1387-1393e1-2.

10. Sutton GP, et al. Ovarian metastases in stage IB carcinoma of the cervix: a Gynecologic Oncology Group study. Am J Obstet Gynecol. 1992;166(1 Pt 1):50-3.

11. Mossa B, et al. Ovarian transposition in young women and fertility sparing. Eur Rev Med Pharmacol Sci. 2015;19(18):3418-25.

12. Baker JW, et al. Preservation of ovarian function in patients requiring radiotherapy for para-aortic and pelvic Hodgkin's disease. Lancet. 1972;1(7764):1307-8.
13. Morice P, et al. Fertility results after ovarian transposition for pelvic malignancies treated by external irradiation or brachytherapy. Hum Reprod. 1998;13(3):660-3.

14. Costa-Roig A, et al. Ovarian transposition as a minimally invasive fertility preservation technique: ten years of experience in a pediatric center. Cir Pediatr. 2020;33(1):25-9.

15. Fernandez-Pineda I, et al. Impact of ovarian transposition before pelvic irradiation on ovarian function among longterm survivors of childhood Hodgkin lymphoma: A report from the St. Jude Lifetime Cohort Study. Pediatr Blood Cancer. 2018;65(9):e27232.

16. Hoekman EJ, et al. Ovarian survival after pelvic radiation: transposition until the age of 35 years. Arch Gynecol Obstet. 2018;298(5):1001-7.

17. Wallace $\mathrm{WH}$, et al. Predicting age of ovarian failure after radiation to a field that includes the ovaries. Int J Radiat Oncol Biol Phys. 2005;62(3):738-44.

18. Wallace WH, Thomson AB, Kelsey TW. The radiosensitivity of the human oocyte. Hum Reprod. 2003;18(1):117-21.

19. Yin L, et al. Ovarian transposition before radiotherapy in cervical cancer patients: functional outcome and the adequate dose constraint. Radiat Oncol. 2019;14(1):100.

20. Morice P, et al. Ovarian transposition for patients with cervical carcinoma treated by radiosurgical combination. FertilSteril. 2000;74(4):743-8.

Publisher's Note Springer Nature remains neutral with regard to jurisdictional claims in published maps and institutional affiliations.

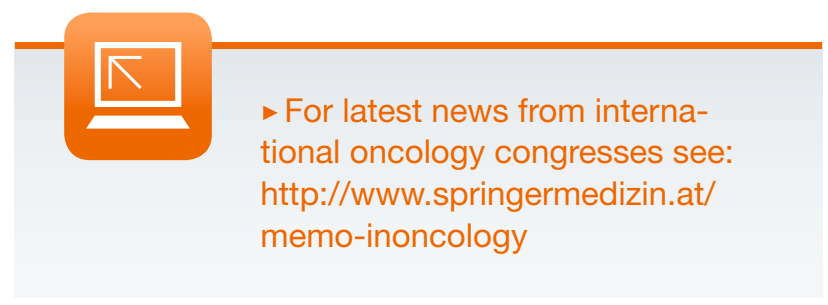

